course, the more accurate its work for the lower degrees. Still I think this is not equal to the triple rotary prisms which I pro. posed, which give the long spaces instead of the short spaces between the low degrees. The Maddox rod, in some form, I rely upon chiefiy for routine examination for heterophoria. I use it in all cases, and if there is reason to follow out that line of investigation further, I afterward resort to other tests of muscular balance.

Dr. H. M. STakkey of Chicago--I used the new instrument of Dr. Prince for a short time, it having been sent to me for study, but not long enough to give any definite idea as to whether I should consider it more useful than other instruments we have. I made, in my mind, the same criticism that Dr. Jackson has spoken of, that it would be more easily used if arranged as Dr. Jackson's adduction prisms are. One point in exercising the adductors; if we use the prisms and find adduction of 10,15 or 25 degrees, we can then place the duction indicator over that and rotate it to the same extent of 15 degrees and the image would still be kept single. If we put a prism of 20 degrees in front of the eyes for stimulating adduction and placing the Prince thorometer in front of that, rotate it until we add 15 degrees, we can not afterward go up to 15 degree with the prisms; it did not stimulate the muscles except in just that way.

Dr. S. D. Risley of Philadelphia-I have been very much interested in this very ingenious instrument and will take an early opportunity to study it more carefully. This is the first I have seen of it in its latest form. I wish to speak particularly of Dr. Carpenter's paper, and to say that I am thoroughly in accord with the principles of classification and the method of examinations to which he has so briefly but clearly referred. I was glad to see him separate those cases that may be due to general dyscrasia. I am sure these may be readily overlooked. There are many persons with rheumatic or gouty diatheses that have transient spells where the amount of variation in the ocular balance is very irregular. We have all seen transient palsy due to gouly disturbance, and before we enter upon operative procedures these cases should be excluded. One class of cases that were not mentioned is the group where abnormalties are simulated by disease at the macula. I have seen cases in which the eyes were drawn this way or that to get rid of an absolute scotoma. If the Maddox rod is used and you guide the patient a little, you will find a blurred spot in the line itself or that a section of the rod is absolutely cut out by the blind spot of the macula. I presented a paper at Edinburgh in which I divided these into two classes, 1 , those due to some anatomic defect in the muscle or its relation to the ball or to disease leading to muscular anomalies, and 2, those due to errors of refraction. The last were relative and disappeared when the refractive error was corrected or with brief training of the mus cles. The patient had to be taught to overcome the relation between convergence and accommodation.

In this group the correcting glasses makeit necessary for him to learn a new order of things as regards the relation between accommodation and convergence, but when he has learned this the relative insufficiencies disappear. I am sure that heterophoria uften exists in consequence of anatomic defects in the skull. We know perfectly well that the skull is distorted in a large number of persons. The first group, or the absolute insufficiencies frequently grow out of these abnormalities inthe skull and shape of the orbit, which give rise to disturbances in the length of the muscles and their attachments to the eyeball and in those cases we may correct the error of refraction, but the abnormality of balance will remain until we have adopted some form of operative interference. These I have called absolute insufficiency.

Dr. J. T. Carpenter, Jr., of Philadelphia-There was one point in my paper in regard to prism exercise for the muscles, which I did not have time to read; that is the value of using the prism exercises after an operation that has not produced sufficient results, I mean immediately after the operation. The result of such exercise has in some cases done permanent good and increased the effect of an operation that was not sufficient; this is especially applicable when the hyperphoria is of low degree and yet a disturbing element. A point that we should not forget is that the entire strength of the vertical muscles is represented by 2 to 4 degrees and a defect of 0.5 degrees even is a serious burden for these muscles.

Dr. A. E. Prince of Springfield -I want to call attention to a point that has occurred to me in regard to discovering a small amount of.hyperphoria that does not render itself apparent upon application of the rod test. It is by sursumduction; in this way, do not commence with the weak prisms and run up, but take a prism that will give you diplopia and then go to weaker ones. That gives the balance and you will find indications that will be quite useful. I will make it clear by a case which had been examined by different men and showed no hyperphoria. I found that if I used a prism that gave diplopia, one of 4 degrees, and then used a 3, 2.5 and 2 degrees prism, and then worked the other eye in the same way, that they come together, one at 2 degrees and one 2.5 degrees, so there was 0.5 degree difference. By putting that correction in the glasses the little patient was entirely relieved of all trouble.

\section{AMBLYOPIA EX ANOPSIA, WITH CASE.}

Presented to the Section on Ophthalmology, at the Forty-eighth Annual Meeting of the American Medical Association, held at Philadelphia, Pa., June 1-4, 1897.

\section{BY A. C. SIMONTON, M.D.} SAN JOSE, CAL.

The only apology that need be offered for presuming to discuss the question of amblyopia ex anopsia before this learned section of the AMERICAN MEDICAL Association is to state the existing fact that in this year 1897 there are eminent ophthalmologists who deny the possibility of the occurrence of the said condition. It would seem that this question should have been definitely settled long since, but it was discussed extensively, pro and con, at the meeting of this AssoCIATION in 1893, and at this meeting we are to have some more pros and cons, no doubt.

How any ophthalmologist who observes closely, thinks clearly and weighs well the terms he is using, will venture to assert that there is no such condition as amblyopia ex anopsia I shall not attempt to explain. While it is a fact that it has not fallen to the good fortune of many oculists to observe the restoration to perfect vision of an eye almost totally blind from disuse, yet mạny ophthalmologists have observed many cases in which there has been partial restoration of vision after a strabismic eye has been rendered par. allel with its fellow. But the restoration not being complete they assert, by the careless line of reasoning and expression before mentioned, that inasmuch as there yet remains amblyopia, the strabismus was caused by original amblyopia and not amblyopia by the strabismus. But when it is acknowledged, as is the case by all authorities, that in a strabismic eye which is highly amblyopic a large percentage of vision may be acquired when the eye is rendered parallel with its fellow and the function of fixation established, is it not equivalent to acknowledging, at least, that the vision thus gained had been in abeyance by reasun of lack of use? If so, does not that constitute amblyopia ex anopsia? If this eye, which gains much vision, had always been parallel with its fellow, does any one suppose for a moment that its visual power would ever have fallen below the full amount now gained by virtue of use in later life? Whether such eye had an original defect which would have rendered it more or less permanently amblyopic or not, it is clear that to the extent vision is restored by use, vision had been lost by virtue of non-use. If one-fourth, one-half or three-fourths of the visual power may be lost or never acquired in a strabismic eye by non-use, is it not logical to conclude that in many cases the total loss in visual power is attributable to this cause? If not logical then why not?

No doubt quite a percentage of eyes become strabismic because of opacities of the cornea, lens or other media, or defective retina, and we find the same class of individuals who deny the existence of amblyopia ex anopsia contend that all strabismic eyes become so by virtue of defect of vision in the converging or diverging eye, and not on account of any conditions relating to accommodation and convergence as 
maintained by Donders. But all agree that there is frequently partial restoration of vision; therefore, there must have been partial amblyopia ex anopsia. And as said before, if part of an amblyopia may be due to want of use the total amblyopia may be thus caused.

The members of our profession who admit fully the existence of amblyopia ex anopsia are also those who agree with Donders that convergent strabismus in a very large majority of cases is due to hypermetropia. The question is raised by those who deny the existence of amblyopia ex anopsia that if convergent strabismus is caused by hypermetropia why do we not have a thousand cases for every one that now exists, since the number of hypermetropes is very great compared with the cases of strabismus? But why are there comparatively so few cases of strabismus when there are so many cases of hypermetropia, has been asked many times and long ago. All students of physiologic optics are familiar with the relations that exist in emmetropia between accommodation and convergence, that these relations are co-ordinate and reciprocal. Now while it is true that under exactly normal conditions there is precisely a given amount of convergence corresponding with a given amount of accommodation, yet these conditions are frequently in abeyance for one reason or another; therefore there is another fortunate possibility on the part of human eyes provided for, and that is the power of dissociating the two functions of convergence and accommodation. All know how this must be done in adult life in the use of optic instruments, and in presbyopia when spectacles are used. But in adult life, after the individual has long enjoyed perfect binocular vision, there has supervened a greater adaptability to varied visual conditions than possibly existed at a time when binocular vision at the near point was being established in infancy.

In a certain percentage of children this power of disassociating the two functions of convergence and accommodation is nil; in other words, they have no relative range of accommodation and convergence. In an infant who can not disasseciate these functions and in whom hypermetropia exists there must inevitably follow convergent strabismus, since accommodation and convergence must be equal. This to my mind is the exact reason why a certain per cent. of hypermetropes become strabismic when accurate binocular vision at close range is being established, and this without the existence of any defect of vision on the part of the converging eye. And again, this theory will explain why it is not necessarily in high degrees of hypermetropia, rather than how, that strabismus occurs.

It is not nearly so difficult to ignore, mentally, an indistinct image in an eye out of parallel with its fellow, as many are given to suppose. Quite occasionally in our tests of muscle equilibrium we find that our patient, although possessed of good binocular vision, can with difficulty recognize the second image produced by the use of prisms.

But when the focal point in a strabismic eye falls upon the disk, as it often does in convergent strabismus, there will be no nervous impression to ignore. It is in these cases of convergent strabismus where the focal point falls on the blind spot that we have early amblyopia, and if conditions are allowed to continue almost total loss of vision in a few years. It is different in divergent strabismus. In this case the focal point falls on sensitive retinæ outside the macula. It has been my observation that in outward deviation the amblyopia is not nearly so great, as a rule, as in the convergent form. In many cases a large amount of vision remains in a divergent eye and yet the person is not troubled with double vision in the least. Close the habitually fixed eye and fairvision will be manifest in the diverging eye. Some of these patients, no doubt, use their eyes alternately, as is the case with many of the lower animals whose eyes are so placed as to preclude binocular fixation. It seems that these animals have the power of acute vision with either eye while ignoring the image in the fellow eye for the time. At all events it remains a fact that in divergent strabismus, in many cases, quite a percentage of vision remains in the divergent eye with no recognized diplopia. But on account of the fact that the focal point does fall upon sensitive retina, although said impression is not recognized in the sensorium, it results in preserving to a greater extent the function of the nervous apparatus of the eye than would be the case did the focal point fall upon the blind spot.

So few have been the cases reported of complete restoration of vision of an eye that has been strabismic and amblyopic that the profession is almost incredulous in regard to such reports. Learned ophthalmologists were surprised by the report of a case by Dr. Johnson at the Milwaukee meeting of this. Association in 1893, in which case there was complete restoration of vision in an eye that had been strabismic and amblyopic from early iafancy, to the extent that vision was almost nil. Some of these able confrères remarked that it was the first and only case. that had come to their notice seeming to establish the doctrine of amblyopia ex anopsia, forgetting, as I have stated before, that partial restoration of vision. of an amblyopic eye is as much evidence of amblyopia ex anopsia as full restoration.

In concluding this paper I will report a case, in my own practice, in which a highly strabismic and amblyopic eye was restored to perfect vision by correcting hypermetropia alone.

Case.-March 31, 1892, Pauline C., aged 8 years, was. brought to my office for consultation about strabismus of the left eye, which had existed from the age of three years. The convergence was in high degree. Owing to the fact that fixation of the left eye had practically always been in abeyance, it was somewhat difficult to assume this function with the right eye covered. Dur first test of vision elicited the information. that the patient could recognize her father at about five feet, and recognized a pencil held in the hand at four feet, but could not tell how many fingers were held before her at this. distance. I attributed this low state of vision on this occasion. to inability to fix the left eye. Vision of right eye was $16 / 60$, and with - spherical 1 D. she read in part $11 \%$. Plus glasses. obscured vision more. Owing to meager vision manifested by the left eye at short range $I$ did not test acuity of vision at. distant letters. I put the eyes under the influence of duboisia for further and better examination. R. V. \$/200. With + S. 2 : D. read $1 \%$. Here was positive evidence that ciliary spasm had existed in this case making an apparent myopia in a case of hypermetropia. Under duboisia and with + S. 2 D. the left. eye now read indistinctly, 11/60. This was the best vision that. could be ohtained with lenses. Skiascopy confirmed the existence of 2 dioptrics of hypermetropia.

I placed $+2.50 \mathrm{~S}$. before these eyes and sent the child home, the eyes boing fully under the influence of duboisia. I hoped on account of the vision remaining in the left eye to be able to overcome the strabismus and acquired amblyopia by glasses alone. The sequel proves that my hope was well founded. It will be observed that I gave over-correction. The: 
patient called at my office April 4,1892, when I discovered the eyes were about parallel. On April 9 strabismus had disappeared. At this time, with + S. 2 D., read $\frac{16}{46}$ with left, showing quite an improvement. I directed that the right eye be covered for two hours each day for distant vision. July 13, three and a half months after adjusting glasses, distant vision of left was $\frac{16}{16}$, and medium print at fourteen inches. I have seen this patient often since the last date and vision is perfect. I have continued, from the first, the use of $2.50 \mathrm{D}$. lenses, both for distance and near. They blurr distant vision slightly, but are no detriment at the near point. My reason for continuing the strong glasses is that the tendency to squint has never disappeared and the instant the glasses are raised from the eyes the left one again converges.

While this was not a case of the most extreme amblyopia, yet it was amblyopia in quite high degree, and it demonstrates most clearly that the strabismus was not due to original amblyopia, for there is none finally left. It demonstrates, also, as clearly as though the case were an extreme one, that the entire amblyopia was due to the strabismus, and if the strabismus had continued a few years longer vision would have been almost totally lost in the left eye. There is nothing clearer to my mind than that this conclusion is correct, and there is such a condition as amblyopia ex anopsia.

\section{AMBLYOPIA FROM SUPPRESSION, CON. GENITAL IMPERFECTION OR DISUSE: WHICH OR ALL?}

Presented to the Section on Ophthalmology, at the Forty-eighth Annual Meeting of the American Medical Association, at Philadelphia, Pa., June 1-4, 1897.

BY LEARTUS CONNOR, A.M., M.D. DETROIT, MICH.

Since different writers use the term "amblyopia ex anopsia" to express radically diverse ideas, it is needful to define its meaning in this discussion. Negatively it is not applied to a dulness of vision induced either by an organic lesion, which can be objectively seen with the ophthalmoscope or otherwise; nor by exclusion of light from the retina, in part or whole, during long periods; nor by defective refraction or disturbed muscular equilibrium of either ciliary, rectus or oblique muscles. Positively it is applied to that dulness of vision which attends the inability of the brain to recognize impressions sent it from the retina.

As to the nature and causes of the dulled vision, we have three widely differing views. The first contends that the brain actively inhibits the visual center from receiving impressions, until it loses its receptive power in whole or part; the second says that the brain or visual center was not properly constructed; while the third argues that the phenomena result from simple disuse, like the disability induced in other organs by a cessation of much or all of their functional activity during a considerable period. The relative importance of these views is of a practical nature. If we adopt congenital imperfection, it matters little whether cases of squint with amblyopia are operated upon early or late; whether the refractive defects be corrected at once or at convenience; the visual results will be the same. If suppression be accepted, then it were criminal to delay in the rectification of those causes which are believed to induce the loss of sight, lest the inhibitory power of the brain continue to the production of absolute blindness. If the amblyopia be regarded as due to simple disuse, then surely every consideration prompts to an immediate restoration of the fullest functional activity.

Ophthalmologic literature affords abundant authority in support of either of these views, and of the practice based thereupon. In this state of the question, discussion by experts is of more than passing moment, and to secure such discussion from this Section is the object of this paper.

The suppression view of amblyopia claims that the brain, being disturbed by conflicting images sent it from separate eyes, inhibits the visual center from recognizing the least perfect one. Frequent repetition of this inhibition, at last, so changes the cell action of the visual centers that they become unable to recognize the imperfect image. These imperfect images are due to a variety of causes, as refracting errors, muscular derangements individually or combined in all sorts of ways. On the suppression view there could be no amblyopia except for imperfect images sent to the visual center and so none without lack of muscular equilibrium, or refractive errors alone or variously combined.

The congenital imperfection view of amblyopia affirms that the visual centers were never normally developed or, if developed, were damaged anterior to birth; it is uncertain whether the defect be in the brain outside or within the visual centers, in the retina or optic nerve fibers. The central scotomas of many cases suggest a pathologic anatomy like that of the toxic amblyopias; they are sometimes for form alone and sometimes for both color and form.

A presumption against the suppression view arises from the fact that in no other instance is it claimed that the brain, by inhibition. actually destroys the functional activity of any organ beyond possibility of restoration. We have many illustrations of imperfectly formed organs, as clubfoot, harelip, colobomas of the iris, retina or choroid, but we know of no organ born with normal development, rendered imperfect by the capricious dislike of the cerebrum.

To determine what light, if any, clinical evidence would throw upon this subject, I studied 7,500 recorded cases of imperfect vision, all from private practice. The standard of amblyopia adopted was an inability to see $20 / 40$ or more after correcting all defective refraction, under full mydriasis, removing all muscular disabilities and securing a normal condition of the general health. Of these above 4,000 were anisometropic, but only sixty were amblyopic; showing that amblyopia was a very infrequent atten. dant of anisometropia, and so unlikely to be caused by it. Anisometropia was found a frequent condition in all cases of convergent squint, but of the fifty-four amblyopic squint cases, only twenty-two were anisometropic. As there was more than three times as many cases of non-amblyopic as of amblyopic squint, it is plain that anisometropia was an unimportant factor in causing amblyopia in the squint cases. Thus my own observations give no reason to believe that anisometropia is a factor in causing amblyopia. According to the suppression view squint is regarded as an important factor in producing amblyopia, while according to the imperfection view, the amblyopia aids in causing squint.

My records do not exhibit a single instance of ambly- 